\title{
Inhibition of Tenebrio molitor antifreeze protein on methane hydrate growth revealed by molecular dynamics simulation
}

\author{
YUE ZHANG, JINGYUE SUN, YINGYING CUI, CONG \\ CHEN AND WEIZHONG LI
}

Dalian University of Technology

Presenting Author: 2962856248@qq.com

Antifreeze protein (AFPs) are biomolecules that are extracted from organisms surviving in freezing conditions and have been regarded as environmental-friendly kinetic ice or hydrate inhibitors. They play essential roles in aspects of oil and gas flow assurance and efficient exploitation of natural gas hydrates. Among them, Tenebrio molitor antifreeze protein(TmAFP), an insect antifreeze protein, was found to have a great effect on inhibition of ice nucleation and growth through previous experiments and simulations, yet its performance as a hydrate inhibitor has not been studied. In this paper, we investigated the inhibition of TmAFP in methane hydrate formation via the molecular dynamics (MD) simulation. Kinetic parameters and the number of hydrogen bonds and water hydrate cages were analyzed. The results showed that TmAFP inhibits the growth of methane hydrate through the so-called adsorption-inhibition mechanism. TmAFP connects to the hydrate surface from THR3 or THR76, and THR-16,28,50,62,76 and ALA-14,74 provide the good shape complementary to replace on the half-cages. It was also found that the non-ice binding surface (NIBS) of the protein includes more hydrogen bonds than ice binding surface (IBS), and its role in hydrate growth was deduced. Our work indicated that TmAFP is a potential hydrate inhibitor with a good application prospect. 